\title{
Efficient Compressive Spectrum Sensing Algorithm for M2M Devices
}

\author{
Zhijin Qin, Yue Gao, Mark D. Plumbley, Clive G. Parini, Laurie G. Cuthbert \\ Queen Mary University of London \\ Electronic Engineering and Computer Science \\ London, United Kingdom \\ \{z.qin, yue.gao, mark.plumbley, c.g.parini, laurie.cuthbert\}@qmul.ac.uk
}

\begin{abstract}
Spectrum used for Machine-to-Machine (M2M) communications should be as cheap as possible or even free in order to connect billions of devices. Recently, both UK and US regulators have conducted trails and pilots to release the UHF TV spectrum for secondary licence-exempt applications. However, it is a very challenging task to implement wideband spectrum sensing in compact and low power M2M devices as high sampling rates are very expensive and difficult to achieve. In recent years, compressive sensing (CS) technique makes fast wideband spectrum sensing possible by taking samples at sub-Nyquist sampling rates. In this paper, we propose a two-step CS based spectrum sensing algorithm. In the first step, the CS is implemented in an SU and only part of the spectrum of interest is supposed to be sensed by an SU in each sensing period to reduce the complexity in the signal recovery process. In the second step, a denoising algorithm is proposed to improve the detection performance of spectrum sensing. The proposed two-step CS based spectrum sensing is compared with the traditional scheme and the theoretical curves.
\end{abstract}

\section{INTRODUCTION}

Recently, Machine-to-Machine (M2M) communications has been identified as a priority area by both the UK and the US as the benefits of M2M are evident in such field as energy, manufacturing, e-health, transportation. As promoted by Federal Communications Commission (FCC) and the UK Office of Communications (Ofcom), TV white space (TVWS) provides an excellent opportunity to enable M2M applications for secondary licence-exempt usage [1][2]. Current approaches to identifying the TVWS rely on a centrally controlled geolocation database. The real-time events and dynamic changes of propagation environment pose significant challenges to the database approach. Therefore, efficient spectrum sensing becomes a natural approach to solving this problem.

In spectrum sensing algorithm, the spectrum of interest can be divided into multiple channels. Only a small portation of spectrum is occupied by primary users (PUs). Traditional spectrum sensing algorithms method allow a secondary user (SU) to sense one narrowband channel only, e.g. maximum $8 \mathrm{MHz}$ for one TV channel or maximum $20 \mathrm{MHz}$ for the $4 \mathrm{G}$ implementation [2][3], in one sensing period. If the narrowband channel is occupied, an SU has to sense another channel in the next period until a vacant channel is found for the SU to transmit signals. It would be more efficient if the SU could sense multiple channels simultaneously in a sensing period. In [4], SUs are divided into a number of nondisjoint feasible subsets in a cooperative spectrum sensing network. Only one subset of SUs is turned on at a period of time and the other nonactivated SUs are in a low-energy sleep mode to make the network energy-efficient.

However, in wideband spectrum sensing, the sampling rates would be very high and difficult for hardware to achieve. As the spectrum is normally underutilized in reality, the spectrum has a sparse property in the frequency domain. This sparse property makes sub-Nyquist rates possible by implementing compressive sensing (CS) technique in SUs [5].

When CS is performed in an SU, only the compressed measurements are collected by the SU. In order to determine the spectrum occupancy, signals should be recovered from those compressed measurements. The common CS recovery algorithms include $l_{1}$ based algorithms such as basis pursuit denoising (BPDN) [6], and greedy iterative algorithms such as thresholding and orthogonal matching pursuit (OMP) [7]. It is noticed that the computational complexity of greedy algorithms is much less than that of $l_{1}$ based algorithms. But greedy algorithms normally require prior information to some extent, such as the sparsity order of signals to be sensed. On the other hand, for $l_{1}$ based algorithms, no prior information of PUs is required but the computational complexity in a recovery process is high and increases with increasing number of samples.

In recent research, CS has been applied to wideband spectrum sensing [8][9][10]. In [8], a matrix completion and joint sparsity recovery based spectrum sensing algorithm is proposed to reduce sensing and transmission requirements and improve sensing results. However, performance of the proposed algorithm is studied under $10 \mathrm{~dB}$ to $45 \mathrm{~dB}$. In [9], a cyclic detector is proposed to reduce the high sampling rates by utilizing the CS principle and exploiting the unique sparsity structure in the two-dimensional cyclic spectrum domain. Its performance is studied though simulations at $\mathrm{SNR}=5 \mathrm{~dB}$. In [10], an efficient COR algorithm with low complexity is proposed for wideband spectrum sensing at SNR $>-10 \mathrm{~dB}$. In these algorithms, prior information of PUs such as sparsity order is considered to be known, which may not be available in practical applications.

In this paper, we propose an algorithm with low complexity, which can handle unknown sparsity order and which is robust to heavy noise. To this end, a two-phase CS based spectrum sensing algorithm for a single node is proposed to reduce the signal recovery complexity and improve detection performance of spectrum sensing. In the first phase, as prior information of sparsity order is unknown, $l_{1}$ based BPDN is utilized to perform signal recovery. Due to the high complexity of the $l_{1}$ 
based algorithm, a new wideband division scheme is proposed to reduce the computational complexity of the signal recovery process by reducing the number of samples to be recovered. In the second phase, after signal is recovered, a denoising algorithm is performed to improve detection performance and enable the algorithm to be more robust to channel noise.

\section{Compressive Sensing Based Spectrum Sensing SYSTEM MODEL}

A four-step CS based spectrum sensing mainly includes sparse representation of received signals, compressed measurements collection, signal recovery and decision making.

\section{A. Sparse representation of the source signals}

It is assumed that the bandwidth of the whole spectrum is $B \mathrm{~Hz}$. The received signal in the $\mathrm{SU}$ is $r(t)=h(t) * s(t)+$ $w(t)$, where $s(t) \in R^{N \times 1}$ is the time domain representation of the transmitted signal, $h(t)$ is the channel gain between the transmitter and receiver, and $w \sim i i d\left(0, \sigma^{2}\right)$ refers to Additive White Gaussian Noise (AWGN).

The frequency domain representation of the received signal $r(t)$ then can be expressed as:

$$
r_{f}=h_{f} s_{f}+w_{f}
$$

where $r_{f}, h_{f}, s_{f}$ and $w_{f}$ are the discrete Fourier transform (DFT) of $r(t), h(t), s(t)$ and $w(t)$. As mentioned above, $s_{f}$ is sparse since the spectrum is normally underutilized. This sparse property makes it possible to reduce sampling rates by implementing CS in the SU.

\section{B. Compressed measurements collection}

After the CS is applied in the SU, the received compressed measurements can be expressed by:

$$
x=\Phi r_{f}=\Phi h_{f} s_{f}+\Phi w_{f}
$$

where $\Phi \in C^{P \times N}(P<N)$ is a Gaussian distributed measurement matrix utilized to collect the compressed measurements $\mathrm{r}_{c} \in C^{P \times 1}, \Phi w_{f} \sim \operatorname{iid}\left(0, \sigma^{2}\right)$ is the noise sample vector as $\Phi$ is column normalized.

\section{Signal recovery}

When CS is performed in the SU, sampling rates are reduced. However, in order to make accurate decision about spectrum occupancy, original signals should be reconstructed before decision making. Signal recovery can be formulated as a convex optimization problem and solved by BPDN as:

$$
\begin{aligned}
& \min \left\|\hat{s}_{f}\right\|_{1} \\
& \text { subject to }\left\|\Phi \cdot \hat{s}_{f}-x\right\|_{2}^{2} \leq \sigma^{2}
\end{aligned} .
$$

\section{Decision making}

When the reconstructed signal $\hat{s}_{f}$ is obtained by solving the above convex problems, energy detection (ED) is performed to determine the spectrum occupancy. The energy of the recovered signals is compared with a predefined threshold to make a decision. The predefined threshold $\lambda_{d}$ is dependent on the noise variance $\left(\sigma^{2}\right)$, the target probability of false alarm $\left(P_{f}\right)$ and the number of samples $\left(N_{\mathrm{O}}\right)$ [11]:

$$
\lambda_{d}=\sigma^{2}\left(1+\frac{Q^{-1}\left(P_{f}\right)}{\sqrt{N_{\mathrm{o}} / 2}}\right) .
$$

If the energy of the reconstructed signal is higher than the threshold, the corresponding channel is determined as occupied by PUs, and SUs are forbidden to access it. Otherwise, the corresponding channel is determined as vacant, and SUs can access it to transmit unlicensed signals.

\section{The Proposed Two-PhASE Compressive SEnsing BAsed Spectrum Sensing Algorithm}

As the prior information about PUs is assumed unknown, $l_{1}$ based recovery algorithm is adopted to perform the signal recovery. However, the computational complexity of $l_{1}$ based methods is higher than greedy algorithms [12]. In order to reduce the complexity in the signal recovery process and enhance the algorithm's robustness to imperfect channel environment, we propose a two-phase CS based spectrum sensing algorithm. In the first phase, a new efficient channel division scheme is proposed to reduce the number of samples to be recovered. In the second phase, a denoising algorithm is proposed to enable the algorithm to be more robust against heavy channel noise.

\section{A. The efficient channel division scheme}

When an $l_{1}$ based algorithm is adopted in an SU, the computational complexity of the signal recovery process is dependent on the number of samples to be recovered. It is assumed that there are $L$ channels in the spectrum of interest. We propose a new channel division scheme in which only $K(K<L)$ channels are expected to be sensed in one sensing period to reduce the number of samples to be recovered. Meanwhile, the necessary sampling rates for the SU are also reduced. As shown in Fig. 1, each $K$-channel group is indexed by $i\left(i=1,2, \cdots, \frac{L}{K}\right)$. After the whole spectrum of interest is divided by the channel division scheme, the CS based single node spectrum sensing process can be summarized as shown in Fig. 2.

Once the signal for the $K$-channel group $s_{f i} \in C^{n \times 1}$ ( $n=\frac{K N}{L}$ ) arrives at the receiver, compressed samples $x_{i}$ are collected at sub-Nyquist sampling rates. Subsequently, the recovered frequency domain representations of the ith $K$ channel group $\hat{s}_{f i}$ can be solved by BPDN as:

$$
\min \left\|\hat{s}_{f i}\right\|_{1} \quad \text { s.t. }\left\|\Phi \cdot \hat{s}_{f i}-x_{i}\right\|_{2}^{2} \leq \sigma^{2} .
$$

\section{B. The denoised spectrum sensing algorithm}

When making a decision for spectrum occupancy, the decision accuracy is influenced by the recovery error of signals. The recovery performance of BPDN is degraded by heavy channel noise and reduced numbers of compressed samples. As a result, the recovery error becomes higher with heavier noise and fewer measurements. Furthermore, it is noticed that the amplitudes of the recovered signal $\hat{s}_{f i}(j)$ may become negative with high absolute values, while the real amplitude values of $\hat{s}_{f i}(j)$ are all processed to be positive. If those negative amplitudes are kept and used to calculate the power of each subband, the power value would becomes higher than the real value which will increase $P_{f}$ which means the vacant 


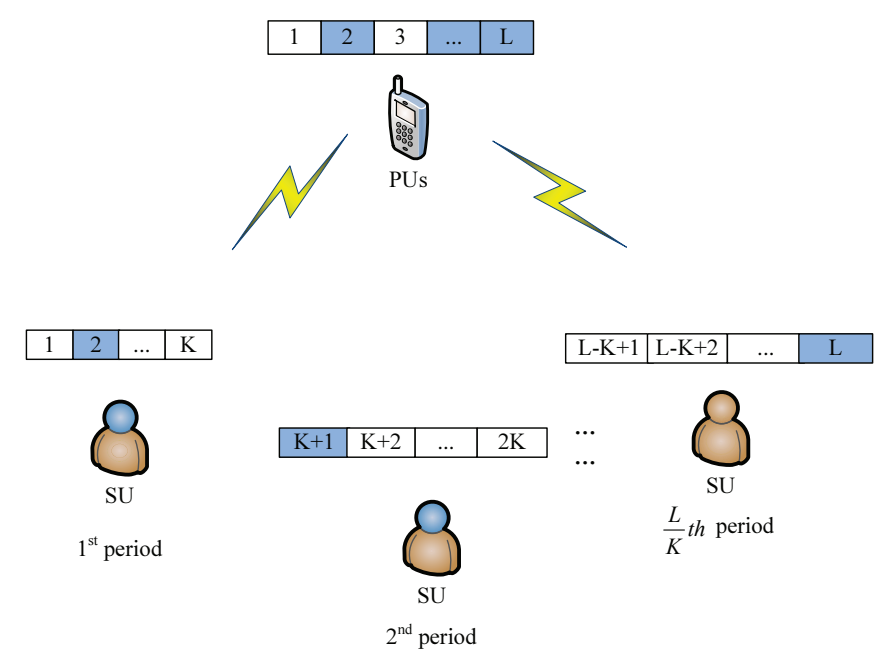

Unoccupied channel

Occupied channel

Fig. 1: Single node spectrum sensing model.

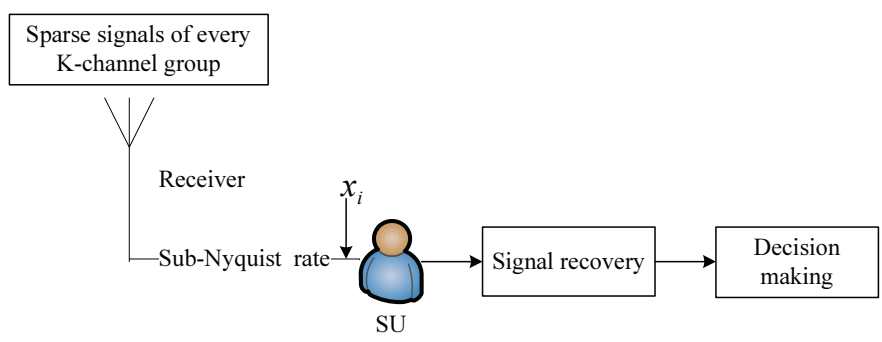

Fig. 2: Process of compressive spectrum sensing.

channel is determined as occupied. In order to improve the recovery performance and detection performance, a denoising algorithm is proposed.

In the denoising algorithm, the amplitudes of $\hat{s}_{f i}(j)$ in the frequency domain are compared with the noise level $\sigma$. If the amplitude of $\hat{s}_{f i}(j)$ is higher than the current noise level, the corresponding amplitude of compressed measurements $r_{f i}(j)$ is kept. Otherwise, the corresponding amplitude will be set to be 0 to reduce the recovery error. The denoised signal $\hat{s}_{f i \_d}$ can be expressed as:

$$
\hat{s}_{f i \_d}(j)=\left\{\begin{array}{cc}
r_{f i}(j) & \text { if } \hat{s}_{f i}(j) \geq \sigma \\
0 & \text { otherwise }
\end{array} .\right.
$$

After denoising algorithm is performed, the energy of the signals is compared with the corresponding threshold under certain $P_{f}$ as defined in (4) to determine the spectrum occupancy of the corresponding $K$-channel group. If the channels are determined as vacant, they can be used by the SU to transmit unlicensed signals. Otherwise, the SU should sense the next $K$-channel group until the vacant channels are found for it to access, or five sensing periods passed. If vacant channels can be found, the SU should firstly sense the $K$ channel group determined as free in last five continuous sensing periods, named as a sensing loop. If no vacant channel can be found in the last sensing loop, the SU should keep sensing from the first $K$-channel group as there is a high probability that the spectrum vacant in last loop keeps to be

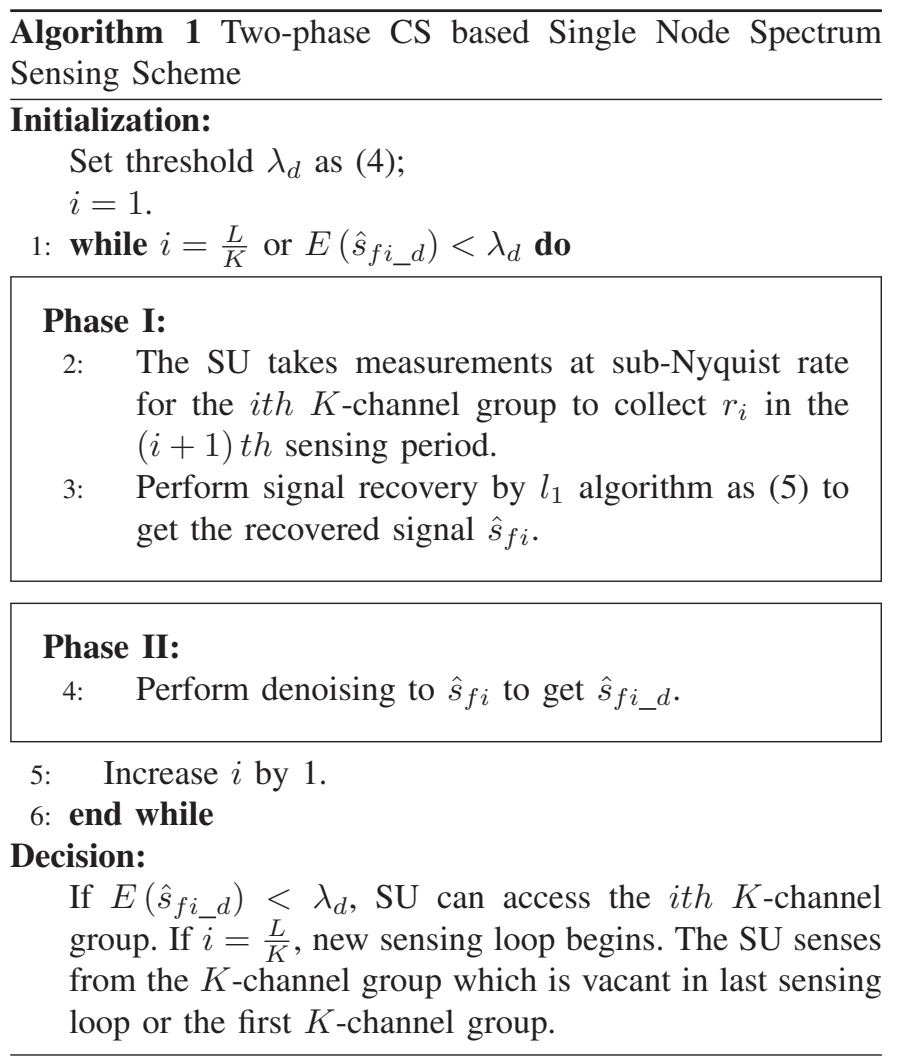

free in the current sensing loop. The whole process of the proposed two-phase CS based spectrum sensing algorithm is summarized as Algorithm 1.

\section{NumericAl Results}

In the simulation, PUs are orthogonal frequency-division multiplexed (OFDM), as used in Digital Video BroadcastingTerrestrial (DVB-T) over the TV white space (TVWS) spectrum from $470 \mathrm{MHz}$ to $790 \mathrm{MHz}$ in the UK [2]. There are 40 channels in total with bandwidth $8 \mathrm{MHz}$ for each channel. It is assumed that the number of channels sensed by the $\mathrm{SU}$ in each sensing period is set to be $8(K=8)$. Therefore, 5 sensing periods are needed to finish the sensing for the whole spectrum of interest. $P_{f}$ is set to be 0.01 . The SNR is defined as the ratio of signal power and noise power of a $K$-channel group.

The computational complexities of (i) spectrum sensing without CS, (ii) CS based spectrum sensing with the whole spectrum sensed, and (iii) CS based spectrum sensing algorithm with the proposed channel division scheme are $O(B)$, $O\left(\frac{B P}{N}\right)$ and $O\left(\frac{K B P}{L N}\right)$ respectively. Since $P$ is much smaller than $N$, we can see that the required sampling rates in the proposed two-phase CS based spectrum sensing are $\frac{K P}{L N}$ times lower than that of spectrum sensing without CS implemented and $\frac{L}{K}$ times lower than that when the whole spectrum of interest is sensed by an $\mathrm{SU}$ in one sensing period.

Fig. 3 shows the $P_{d}$ of the proposed two-phase CS based single node spectrum sensing algorithm (labelled as denoised BPDN) under different compression ratios. Its detection performance is also compared with that of spectrum sensing algorithm without CS implemented, as well as the theoretical values derived from [13] [14]: 


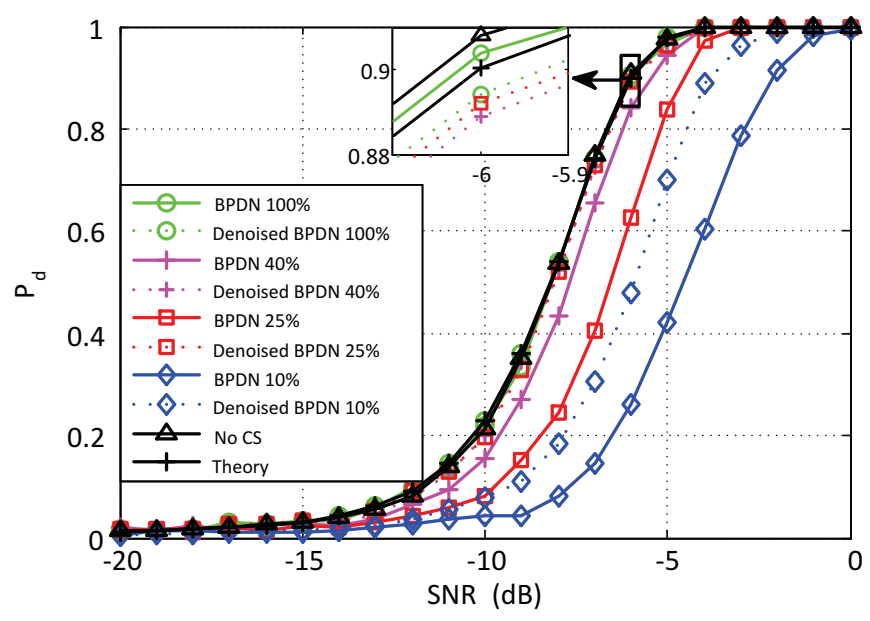

Fig. 3: $P_{d}$ comparison with different compression ratios.

$$
P_{d}=Q\left(\frac{\lambda_{d} / \sigma^{2}-(1+\mathrm{SNR})}{(1+\mathrm{SNR}) / \sqrt{N_{\mathrm{O}} / 2}}\right) .
$$

When the compression ratio is $100 \%$, the signal recovery is exact. Fig. 3 shows that the performance of BPDN based spectrum sensing algorithms with and without denoising are both the same with that of algorithm without CS implemented in the SU and the theoretical curves obtained by (7). When the number of available samples decreases, the detection performance degrades. It also shows that the performance of the proposed two-phase CS based spectrum sensing is better than that of the CS based spectrum sensing without denoising when the compression ratio is $40 \%, 25 \%$ and $10 \%$. This gain benefits from the denoising algorithm, which can improve the signal recovery accuracy. As the recovery accuracy becomes higher with the higher compression ratio, the detection performance of the proposed two-phase CS based spectrum sensing algorithm gets closer to the theoretical curves. The simulation result shows that the proposed two-phase CS based spectrum sensing algorithm can let the sampling rates reduce by $75 \%$ without degrading detection performance.

Fig. 4 shows the detection performance of the proposed two-phase CS based spectrum sensing algorithm (labeled as denoised BPDN) with different numbers of active PUs in an 8-channel group. In this scenario, the compression ratio is set to $25 \%$. The number of active PUs changes from 1 to 3 in an 8-channel group, which means the sparsity order of the signal to be recovered changes from $12.5 \%$ to $37.5 \%$. The positions of these active PUs are set to be random. The detection performance becomes worse with increasing sparsity order as shown in the figure. As the sparsity order increases, the sparse property of the signals to be recovered becomes worse. As a result, when the sparsity order increases, more compressed measurements should be collected for signal recovery to make sure the detection performance is not degraded. But we can see that the detection performance would only be slightly degraded when the proposed algorithm is applied to the practical sensing for TVWS spectrum as its occupancy ratio is normally $15 \%$ to $20 \%$ in practice [1][2].

The Receiver Operating Characteristics (ROC) curves under different SNR values are shown in Fig. 5, where the

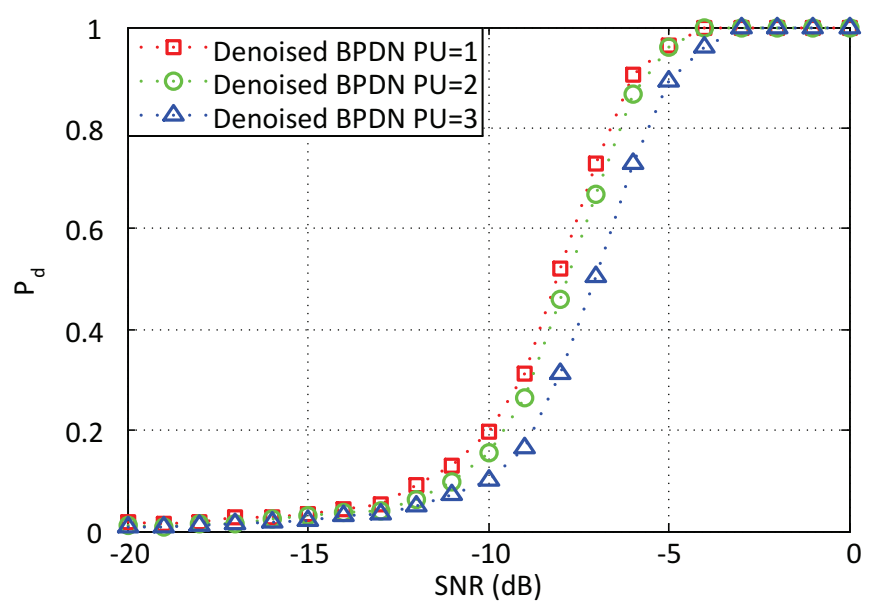

Fig. 4: $P_{d}$ comparison with different number of active PUs.

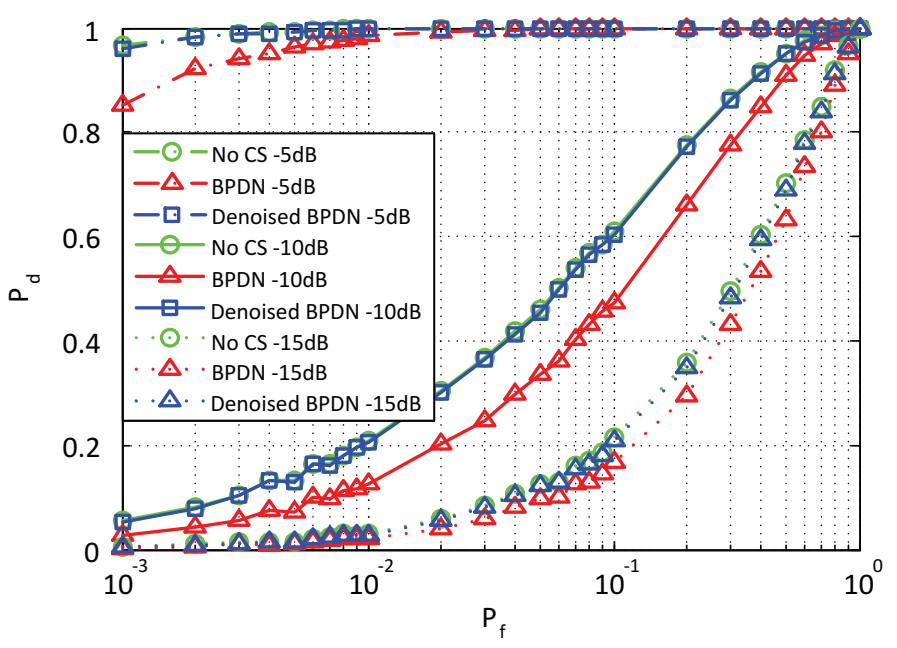

Fig. 5: Comparative ROC curves under different SNR values, compression ratio is $25 \%$.

compression ratio is set to be $25 \%$. It can be observed that the proposed two-phase CS based spectrum sensing (labelled as denoised BPDN) exhibits better performance than the CS based spectrum sensing without the denoising algorithm implemented. It is also noticed that the performance of the CS based spectrum sensing algorithms is almost as good as that of spectrum sensing without CS. This gain benefits from the denoising algorithm. This result matches with that in Figure. 3 when the compression ratio is set to be $25 \%$.

\section{CONCLUSions}

In wideband spectrum sensing, high sampling rates are difficult to achieve, especially for power limited M2M devices. The CS technique could be implemented in SUs to achieve sub-Nyquist sampling rates. In this paper, a two-step CS based spectrum sensing algorithm is proposed to reduce the complexity in the signal recovery process and improve the detection performance of spectrum sensing. The simulation showed that the detection probability of our proposed twostep CS based spectrum sensing outperformed the traditional scheme with fewer measurements. Therefore, the proposed algorithm can be a strong contender for M2M devices. 


\section{REFERENCES}

[1] P. Kolodzy and I. Avoidance, "Spectrum policy task force," Federal Commun. Comm., Washington, DC, Rep. ET Docket, no. 02-135, 2002.

[2] UK Office of Communications (Ofcom), Statement on Cognitive Access to Interleaved Spectrum, Jul, 2009.

[3] 3GPP and TS36.300, "Evolved universal terrestrial radio access (EUTRA) and evolved universal terrestrial radio access network (EUTRAN); overall description," Jul. 2013.

[4] R. Deng, J. Chen, C. Yuen, P. Cheng, and Y. Sun, "Energy-efficient cooperative spectrum sensing by optimal scheduling in sensor-aided cognitive radio networks," IEEE Transactions on Vehicular Technology, vol. 61, no. 2, pp. 716-725, Feb 2012.

[5] E. Candes, "Compressive sampling," in Proceedings of the International Congress of Mathematicians, vol. 3, Madrid, 2006, pp. 1433-1452.

[6] S. S. Chen, D. L. Donoho, and M. A. Saunders, "Atomic decomposition by basis pursuit," SIAM Journal on scientific computing, vol. 20, no. 1, pp. 33-61, Aug. 1998.

[7] Y. C. Pati, R. Rezaiifar, and P. Krishnaprasad, "Orthogonal matching pursuit: Recursive function approximation with applications to wavelet decomposition," in 27th Asilomar Conference on Signals, Systems and Computers, Pacific Grove, CA, 1993, pp. 40-44.

[8] J. Meng, W. Yin, H. Li, E. Hossain, and Z. Han, "Collaborative spectrum sensing from sparse observations in cognitive radio networks," IEEE Journal on Selected Areas in Communications, vol. 29, no. 2, pp. 327337, Feb. 2011.

[9] Z. Tian, Y. Tafesse, and B. M. Sadler, "Cyclic feature detection with sub-Nyquist sampling for wideband spectrum sensing," IEEE Journal of Selected topics in signal processing, vol. 6, no. 1, pp. 58-69, Feb. 2012.

[10] S. Mistry and V. Sharma, "New algorithms for wideband spectrum sensing via compressive sensing," in IEEE International Conference on Communications (ICC), Budapest, 2013, pp. 2595-2600.

[11] F. Digham, M.-S. Alouini, and M. K. Simon, "On the energy detection of unknown signals over fading channels," in IEEE International Conference on Communications (ICC), Anchorage, 2003, pp. 3575-3579.

[12] J. Tropp and A. Gilbert, "Signal recovery from random measurements via orthogonal matching pursuit," IEEE Transactions on Information Theory, vol. 53, no. 12, pp. 4655-4666, Dec. 2007.

[13] Z. Ye, G. Memik, and J. Grosspietsch, "Energy detection using estimated noise variance for spectrum sensing in cognitive radio networks," in IEEE Wireless Communications and Networking Conference (WCNC), Las Vegas, NV, 2008, pp. 711-716.

[14] N. Wang, Y. Gao, and X. Zhang, "Adaptive spectrum sensing algorithm under different primary user utilizations," IEEE Communications Letters, vol. 17, no. 9, pp. 1838-1841, Sep. 2013. 